American Medical Journal 3 (1): 43-46, 2012

ISSN 1949-0070

(C) 2012 Science Publications

\title{
Childhood Mental Disorders and Risk of Schizophrenia
}

\author{
Wenbin Liang and Tanya Chikritzhs \\ National Drug Research Institute, Health Science, \\ Curtin University, Perth, Western Australia, Australia
}

\begin{abstract}
Problem statement: Schizophrenia is one of the most serious forms of mental diseases, but the etiology of schizophrenia is still not well understood. Investigations are required to gain better understanding of the pre-onset course of schizophrenia. Approach: This study was a populationbased birth-cohort study of males born between 1980 and 1984 in Western Australia (WA). Males were identified using birth registry records and followed-up until December 31st, 2009 or death using linked health data available through the Western Australian Data Linkage System (WADLS). Results: Children who developed a mental disorder before the age of $12 \mathrm{yrs}$ had about at twice the risk of developing schizophrenia compared to those without a diagnosis. Increased risks appeared to be greatest for specific delays in development and hyperkinetic syndrome of childhood. Conclusion: Children with childhood mental disorders are at higher risk of being diagnosed with schizophrenia later in life. These findings provide further evidence to support the hypothesis that schizophrenia is a neurodevelopmental disorder.
\end{abstract}

Key words: Schizophrenia, hyperkinetic syndrome, cohort study, life course

\section{INTRODUCTION}

Schizophrenia is one of the most serious forms of mental diseases affecting about $1 \%$ of the general population worldwide (Maki et al., 2005) and it has been estimated to account for $2.3 \%$ of the total burden of disease in developed countries (Hales et al., 2008). As a chronic disease, schizophrenia affects not only the life of patients but also poses significant burden on close family members (Hales et al., 2008; McGrath et $a l ., 2008)$. The consensus view among the literature appears to be that schizophrenia is a neurodevelopmental disorder (Fatemi and Folsom, 2009; Pearce, 2001; Murray and Lewis, 1987; Omranifard and Asadollahi, 2009), however the etiology of schizophrenia is still not well understood (Hales et al., 2008; Walker et al., 2004; Jayyab, 2010). One of the weaknesses of the neurodevelopmental model is that there is a long time gap between neurodevelopment which occurs during the prenatal and early childhood periods and the onset of schizophrenia symptoms, which tend to occur in the 20s (Hales et al., 2008; Fatemi and Folsom, 2009; Girolamo et al., 2012). However, it is possible that abnormal neurodevelopment may lead to other detectable mental and psychical abnormalities before the onset of schizophrenia. A few studies have identified signs of mental and psychological deficits before the onset of schizophrenia. It has been observed that children who later developed schizophrenia were more likely to have poorer progress in psychological and cognitive functions at ages 13-16 yrs (Jones et al., 1994). A recently published New Zealand study, suggested that schizophrenia patients may encounter developmental deficits at ages as young as 7-13 yrs (Reichenberg et al., 2010). It was also reported that children with schizophrenia outcome demonstrated abnormal social behaviors and showed less positive emotion (Walker et al., 2004; Walker et al., 1993). In these previous studies, most of the psychological and cognitive abnormalities observed among children who later developed schizophrenia were mild or moderate and did not reach the severity required for clinical diagnosis (Walker et al., 2004). In order to gain better understanding of the preonset course of schizophrenia, this study was conducted to investigate the association between diagnosed childhood mental disorders (0-12 yrs) and schizophrenia among an Australian male population.

\section{METERIALS AND METHODS}

This study was a population-based birth-cohort study of males born between 1980 and 1984 in Western Australia (WA). Males were identified using birth

Corresponding Author: Wenbin Liang, National Drug Research Institute, Curtin University, GPO Box U1987, Perth WA 6845 Australia Tel: +61 892661617 Fax: +61 892661611 
registry records and followed-up until December 31st, 2009 or death using linked health data available through the Western Australian Data Linkage System (WADLS) (Holman et al., 1999).

Demographics, hospitalization and outpatient visit records of mental health conditions before the age of $12 \mathrm{yrs}$ and diagnosis of schizophrenia in outpatient or inpatient psychiatric service during the follow-up period were identified for the birth cohort from three databases and linked by the WADLS. The youngest age at diagnosis of schizophrenia in this birth cohort was 12 yrs (one participant only). Data sources included: (i) Midwives Notifications Data, (ii) mental health service records from the WA Mental Health Ambulatory Data System; (iii) WA hospital morbidity data system; (iv) Birth Registry data; and (v) Mortality Data. The WA hospital morbidity Data System included separations from all WA public and private acute hospitals (Holman et al., 1999). The mental health service records and the WA Hospital Morbidity Data System included all WA public and private psychiatric hospitals and all attendances at public psychiatric outpatient clinics (Holman et al., 1999). The reliability and quality of these linked data have been confirmed previously (Holman et al., 1999). In the Hospital Morbidity Data and the Mental Health Ambulatory Data, diagnosis of mental disorder was mainly coded using ICD-9 (only one diagnosis of mental disorder was coded in ICD-10) during the exposure period (19801996). Both ICD-9 codes and ICD-10 codes were used for some periods of the follow-up and therefore both ICD-9 codes (295) and ICD-10 codes (F20) were used to identify diagnosis of schizophrenia.

To control for potential confounding effects of socio-economic status, WA residential location (Local Government Area, LGA) at birth was coded using the Australia Bureau of Statistics' (ABS) Index of Relative Socio-Economic Disadvantage (IRSED, a general measure of economic disadvantage of residential families in an area) (McLennan, 1998).

Statistical analysis: In the analysis we first assessed the association between all diagnosed mental disorders occurring from 0-12 yrs of age and risk of schizophrenia and then we separately assessed the relation for the six most common groups of mental disorders before 12yrs, specifically: (1) disturbance of emotions specific to childhood and adolescence (ICD-9 313); (2) disturbance of conduct (ICD-9 312); ( 3 ) adjustment reaction (ICD-9 309); (4) specific delays in development i.e. (developmental speech or language disorder) (ICD-9 315); (5) special symptoms or syndromes, not elsewhere classified (i.e. encopresis) (ICD-9 307); and (6) hyperkinetic syndrome of childhood (ICD-9 314). Univariate analysis was first used to estimate the unadjusted association between childhood mental disorders and schizophrenia. Multivariate logistic regression was then used to control for a number of potential confounders: ages of mother and father at delivery; Apgar score at five minutes; whether the mother previously had any negative birth outcomes (miscarriage, death at birth or still birth); and IRSED for residential location at birth. Participants with missing information in any of the control variables were excluded from the multivariate analysis. In order to control for potential confounding effects due to abnormal prenatal developments, newborns with birth weight less than 2,500 g, an Apgar score at five minute less than 7, or with a father or mother younger than 18 yrs at birth were excluded from analysis.

Ethics: Ethical approval for this study was obtained from the Department of Health, Government of Western Australia, Human Research Ethics Committee (DOHWA HREC).

\section{RESULTS}

There were 51464 participants included in the univariate analysis. Some $3.37 \%$ (1736) of the 51464 participants had one or more mental disorder diagnosis before the age of 12yrs. The prevalence of schizophrenia among participants without a mental disorder diagnosis was about 1\%. 45291 participants were included in the multivariate analysis. The estimations from univariate logistic regression models were similar to those from multivariate logistic regressions, indicating negligible confounding effects from the control variables tested. Overall, children with one or more diagnosis of mental disorder before the age of 12 yrs were more likely to develop schizophrenia in later life. When the analysis was performed separately by type of disorder, it was found that disturbance of emotions, specific delays in development and hyperkinetic syndrome of childhood were all significantly associated with increased risk of schizophrenia. The point estimation was highest for delays in development, which indicated a 4.7 times risk of schizophrenia. There was no significant increase in the risk of schizophrenia observed among children with disturbance of conduct, adjustment reaction or special symptoms or syndromes (Table 1). 
Am. Med.J. 3 (1): 43-46, 2012

Table 1: Association between childhood mental disorders and risk of schizophrenia

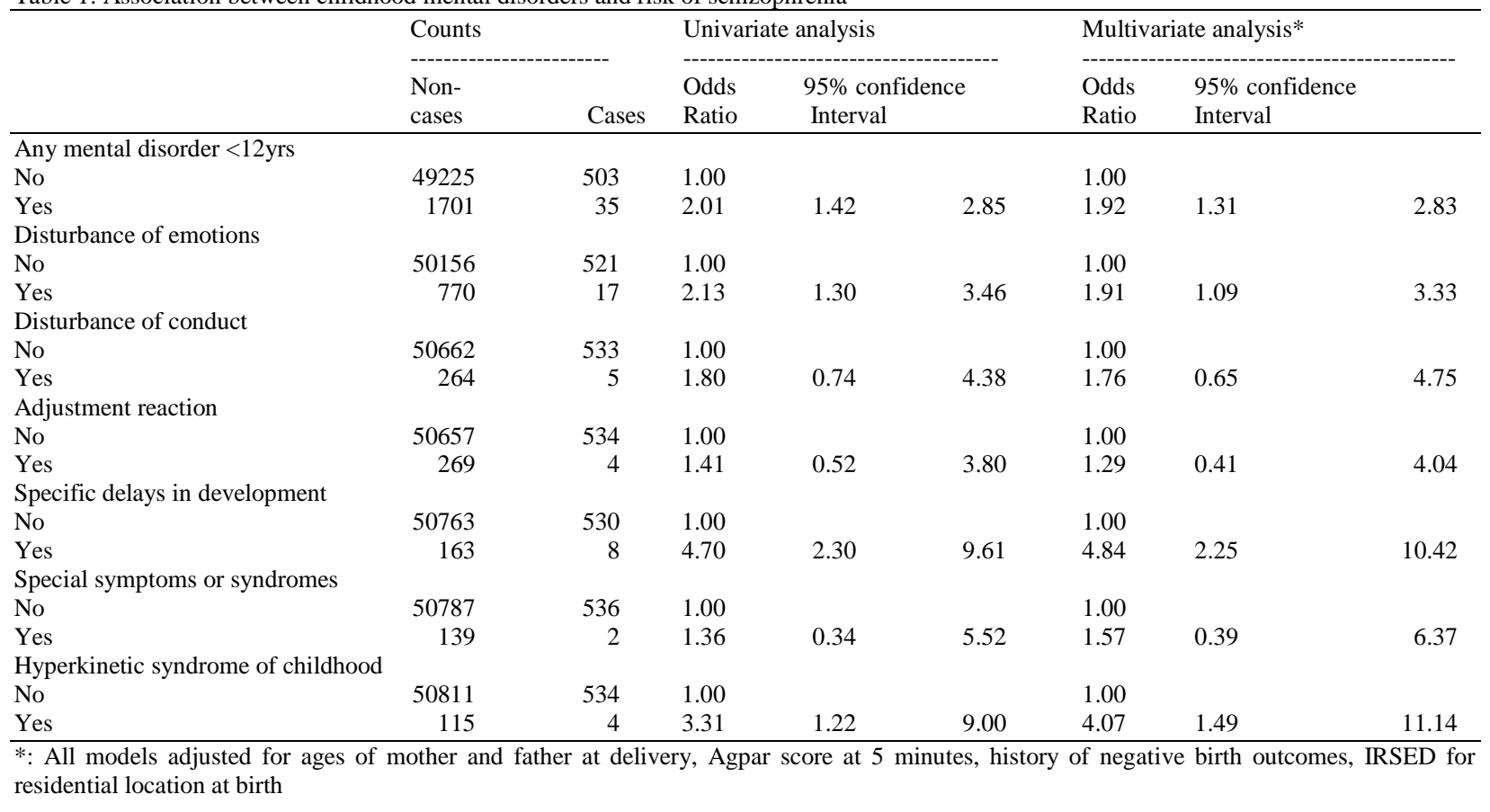

\section{DISCUSSION}

This study investigated the association between mental disorders during childhood and onset of schizophrenia in later life among males with an Australian population-based birth cohort. Childhood mental disorder history and schizophrenia status were both determined by diagnoses made in clinical records. Findings of the current study suggest that children who developed a mental disorder before the age of $12 \mathrm{yrs}$ had about at twice the risk of developing schizophrenia compared to those without a diagnosis. Increased risks appeared to be greatest for specific delays in development and hyperkinetic syndrome of childhood. Non-significant increases were observed in three subgroups of childhood mental disorders, however, it is possible that the small numbers of cases (low population prevalence) reduced the power of the analysis to detect a significant difference.

Overall observations in the current study remained consistent with the findings from previous studies which showed that children developed schizophrenia in later life were more likely to show abnormalities in cognitive and psychological development during childhood (Walker et al., 2004; 1993; Jones et al., 1994, Reichenberg et al., 2010, Crow et al., 1995; Bearden et al., 2000; Poulton et al., 2000). These findings provide further evidence supporting that hypothesis that schizophrenia is a neurodevelopmental disorder. However it should be noted that majority of the children with diagnosed mental disorders during childhood did not develop schizophrenia and similarly, majority of children have abnormalities in cognitive and psychological development did not develop schizophrenia in later life (Jones et al., 1994; Poulton et al., 2000; Cannon et al., 2002). It is possible that the childhood mental disorders among children with schizophrenia outcome may have different underlying causes and brain development deficits compared to children without schizophrenia outcome. This requires further investigation with latest neuroimaging technology, genetic tests and close follow-up, because early detection may ultimately improve outcomes for those with an elevated risk of schizophrenia (Walker et al., 2004; Nan et al., 2008).

Limitations: The main limitation of this study is that it only includes males so the association observed should be further tested in a population-based female cohort. The sample size of the current study is relatively large. However, given that both schizophrenia and childhood mental disorders are relatively uncommon conditions, the subgroup analysis did not have sufficient statistical power for all disorders. To improve efficiency, a population-based nested case-control design can be adopted in future studies. 


\section{CONCLUSION}

Children with childhood mental disorders are at higher risk of being diagnosed with schizophrenia later in life. These findings provide further evidence to support the hypothesis that schizophrenia is a neurodevelopmental disorder.

\section{REFERENCES}

Bearden, C.E., I.M. Rosso, J.M. Hollister, L.E. Sanchez and T. Hadley et al., 2000. A prospective cohort study of childhood behavioral deviance and language abnormalities as predictors of adult schizophrenia. Schizophrenia Bull., 26: 395-410.

Cannon, M., A. Caspi, T.E. Moffitt, H. Harrington and A. Taylor et al., 2002. Evidence for earlychildhood, pan-developmental impairment specific to schizophreniform disorder: Results from a longitudinal birth cohort. Arch Gen Psychiatry, 59: 449-456. PMID: 11982449

Crow, T., D.J. Done and A. Sacker, 1995. Childhood precursors of psychosis as clues to its evolutionary origins. Eur. Arch. Psychiatry Clin. Neurosci., 245: 61-69. PMID: 7654790

Fatemi, S.H. and T.D. Folsom, 2009. The neurodevelopmental hypothesis of schizophrenia, revisited. Schizophr. Bull., 35: 528-548. DOI: 10.1093/schbul/sbn187

Girolamo, G.D., J. Dagani, R. Purcell, A. Cocchi and P.D. McGorry, 2012. Age of onset of mental disorders and use of mental health services: needs, opportunities and obstacles. Epidemiol. Psychiatric Sci., 21: 47-57. DOI: 10.1017/S2045796011000746

Hales, R.E., S.C. Yudofsky and G.O. Gabbard, 2008. The American Psychiatric Publishing Textbook of Psychiatry. 5th Edn., American Psychiatric Publishing, Washington, DC., ISBN: 1585622575 , pp: 1786.

Holman, C.D., A.J. Bass, I.L. Rouse and M.S. Hobbs, 1999. Population-based linkage of health records in Western Australia: Development of a health services research linked database. Aust. N. Z. J. Publ. Health, 23: 453-459. PMID: 10575763

Jayyab, A.A., 2010. Taurine implicated in bromocriptine induced hallucination: Glycineglutamic-aspartic implicated in bromocriptine induced schizophrenia. Am. J. Pharmacol. Toxicol., 5: p. 34-41. DOI: 10.3844/ajptsp.2010.34.41
Jones, P., B. Rodgers, R. Murray and M. Marmot, 1994. Child development risk factors for adult schizophrenia in the British 1946 birth cohort. Lancet, 344: 1398-1402. PMID: 7968076

Maki, P., J. Veijola, P.B. Jones, G.K. Murray and H. Koponen et al., 2005. Predictors of schizophrenia-a review. Br. Med. Bull., 73-74: 1-15. PMID: 15947217

McGrath, J., S. Saha, D. Chant and J. Welham, 2008. Schizophrenia: A concise overview of incidence, prevalence and mortality. Epidemiol. Rev., 30: 6776. DOI: $10.1093 /$ epirev/mxn001

McLennan, W., 1998. 1996 Census of Population and Housing: Selected Family and Labour Force Characteristics, Australia. 1st Edn., Australian Bureau of Statistics, Canberra pp: 106.

Murray, R.M. and S.W. Lewis, 1987. Is schizophrenia a neurodevelopmental disorder? BMJ., 295: 681-682. DOI: $10.1136 / \mathrm{bmj} .295 .6600 .681$

Nan, F., Y. Wang and X. Ma, 2008. Application of multiscale hidden markov modeling wavelet coefficients to fMRI activation detection. J. Math. Stat., 4: 255-263. DOI: 10.3844/jmssp.2008.255.263

Omranifard, V. and G. Asadollahi, 2009. Association between paternal age at birth time and the risk of offspring developing schizophrenia. Am. J. Applied Sci., 6: 179-181. DOI: 10.3844/ajassp.2009.179.181

Pearce, B.D., 2001. Schizophrenia and viral infection during neurodevelopment: A focus on mechanisms. Mol. Psychiatry, 6: 634-646. DOI: 10.1038/sj.mp.4000956

Poulton, R., A. Caspi, T.E. Moffitt, M. Cannon and R. Murray et al., 2000. Children's self-reported psychotic symptoms and adult schizophreniform disorder: A 15-year longitudinal study. Arch. Gen. Psychiatry, 57: 1053-1058. PMID: 11074871

Reichenberg, A., A. Caspi, H. Harrington, R. Houts and R.S. Keefe et al., 2010. Static and dynamic cognitive deficits in childhood preceding adult schizophrenia: A 30-year study. Am. J. Psychiatry, 167: 160-169. PMID: 20048021

Walker, E., L. Kestler, A. Bollini and K.M. Hochman, 2004. Schizophrenia: Etiology and Course. Ann. Rev. Psychol., 55: 401-430. PMID: 14744221

Walker, E.F., K.E. Grimes, D.M. Davis and A.J. Smith, 1993. Childhood precursors of schizophrenia: Facial expressions of emotion. Am. J. Psychiatry, 150: 1654-1660. PMID: 8214173 